\title{
Aplicación de dos Modelos de Simulación Integral Hidrológica, para la estimación de caudales medios mensuales, en dos cuencas de Chile central*
}

\author{
Application of two integral hydrologic simulation models, for the average monthly
}

flow estimation, in two basins of Central Chile

\author{
ROBERTO PIZARRO T. ${ }^{1}$, MANUEL SOTO B. ${ }^{2}$, CESAR FARIAS D. ${ }^{1}$, CRISTIAN JORDAN D. ${ }^{1}$ \\ ${ }^{1}$ Facultad de Ciencias Forestales, Universidad de Talca, Av. Lircay s/n, Talca, Chile. \\ E-mail: rpizarro@utalca.cl \\ ${ }^{2}$ Centro Internacional del Agua para Zonas Aridas y Semiáridas de América Latina y el Caribe (CAZALAC)/ \\ PHI-UNESCO, Cisterna s/n esq. Anfión Muñoz, La Serena, Chile.
}

\section{SUMMARY}

Two rainfall-runoff models, Témez Model and T Model, were calibrated for Purapel river basin and Achibueno river basin, at Maule region of Chile. Although $\mathrm{T}$ Model uses two parameters and Témez Model uses four parameters, more parameters were used in this research by clustering months from a temporal point of view. On the other hand, $66 \%$ of data were used for calibration and the rest for validation. Results show total data variation is explained in $82 \%$ on Purapel river basin through Témez Model and $61 \%$ is explained for Achibueno river basin through T Model. In addition, U Mann Whitney test is used to fit quality. Both models are considered to be good models. Finally, to use Témez Model for Purapel river basin and T Model for Achibueno river basin is suggested.

Key words: rainfall-runoff Models, simulation Models, Témez Model, T Model.

\section{RESUMEN}

Se calibraron y validaron dos modelos de simulación integral de cuenca, Modelo T (de 2 parámetros) y Modelo Témez (de 4 parámetros), para las cuencas del río Purapel $\left(264.6 \mathrm{~km}^{2}\right)$, y del río Achibueno $\left(943 \mathrm{~km}^{2}\right)$, respectivamente, en la Región del Maule, Chile. La calibración consideró el planteamiento original de los modelos, pero diferenciando parámetros para distintos meses o grupos de meses, llegando a establecer 8 parámetros en el río Purapel y 24 parámetros en el río Achibueno. La validación se analizó a base de pruebas estadísticas no paramétricas. Así, los resultados muestran que un $82 \%$ de aportación específica de la cuenca del río Purapel es explicada por el modelo Témez, mientras que un $61 \%$ de la aportación de la cuenca del río Achibueno es explicada por el modelo T. Se recomienda el Modelo Témez de 8 parámetros para la cuenca del río Purapel y el Modelo T de 24 parámetros para la cuenca del río Achibueno, como herramienta para la evaluación del recurso hídrico, ya que incluyen los principales procesos del ciclo hidrológico en su simulación y presentan un número de parámetros manejables.

Palabras clave: modelos de simulación, simulación integral de cuencas, modelo T, modelo Témez.

* Esta investigación se enmarca dentro del proyecto FONDECYT 1010590. 
BOSQUE 26(2): 123-129, 2005

Aplicación de dos Modelos de Simulación Integral Hidrológica

\section{INTRODUCCION}

Las relaciones hidrológicas que ocurren en una cuenca se pueden estudiar a través de modelos que simplifiquen y representen los distintos fenómenos que suceden al interior de ésta, siendo los modelos matemáticos una herramienta importante y que se ha ido perfeccionando vertiginosamente durante el siglo XX. Así, los modelos matemáticos permiten representar un sistema hidrológico por medio de relaciones lógicas y cuantitativas, capaces de ser modificadas para observar cómo el sistema reacciona, siendo los modelos de simulación aquellos capaces de reproducir sistemas altamente complejos (1). Debido a la complejidad de los procesos hidrológicos que ocurren en un sistema natural, la ingeniería hidrológica ha desarrollado modelos que en términos generales reproducen el ciclo hidrológico o parte de éste, basados en el supuesto que un modelo general puede ser modificado para utilizarse en otras cuencas de características similares $(2,3,4,5)$.

En este contexto, los Modelos de Simulación Integral de Cuencas (MSIC) representan la totalidad del ciclo hidrológico que ocurre en una cuenca hidrográfica, teniendo como principal finalidad generar datos de caudal o aportación, a partir de información meteorológica (principalmente precipitaciones y evapotranspiraciones potenciales (ETP)), los cuales pueden someterse a análisis probabilísticos para la determinación de parámetros en el diseño de obras hidráulicas. Además, son capaces de producir datos en cuencas sin estaciones de medición y permiten realizar la completación y extensión de series históricas de aportaciones $(4,5,6)$.

El desarrollo de estos métodos indirectos de evaluación de los recursos hídricos, sumado a los avances computacionales y a la masificación de los sistemas de información geográfica (SIG), han permitido el análisis de los procesos hidrológicos considerando intervalos de tiempos más cortos y con un mayor detalle espacial dentro de la cuenca (1).

La presente nota técnica muestra los resultados de dos Modelos de Simulación Integral de Cuencas (MSIC), Modelo T (3) y Modelo Témez $(4,5)$, aplicados en las cuencas del río Purapel con una extensión de 264,6 km², y del río Achibueno de $943 \mathrm{~km}^{2}$, ubicados ambos en la Región del Maule en la zona central de Chile. Para ello, se realizaron la calibración y validación, a nivel mensual, de los dos modelos de simulación, los que fueron seleccionados por la poca cantidad de parámetros necesarios para su aplicación, además por su utilidad en la hidrología forestal, ya que permiten comparar el comportamiento de los caudales en distintas condiciones de cubierta vegetal y permiten generar datos en cuenca sin información, con lo cual se transforman en una potente herramienta para el diseño de obras hidráulicas en la planificación de faenas forestales.

\section{DESCRIPCION DEL AREA DE ESTUDIO}

La cuenca del río Purapel se localiza en el secano interior de la Cordillera de la Costa; presenta una superficie de $264,6 \mathrm{~km}^{2}$ (figura 1), entre el origen y la estación de aforo Purapel en Nirivilo, de propiedad de la Dirección General de Aguas (DGA). Sus cotas mínimas y máximas, en esta superficie, varían entre los 96 y $540 \mathrm{~m}$ snm, respectivamente. La orientación del cauce principal va de oeste a suroriente, con una longitud del cauce principal de $25 \mathrm{~km}$. Posee un régimen hidrológico netamente pluvial, presentando una precipitación media anual, entre 1969 y 2000, de $812,4 \mathrm{~mm}$.

La cuenca del río Achibueno se ubica en la Cordillera de los Andes, tiene una superficie de $943 \mathrm{~km}^{2}$, entre el origen y la estación de aforo Achibueno en La Recova, perteneciente a la DGA (figura 1). Sus cotas varían entre los $1.810 \mathrm{y}$ $590 \mathrm{~m}$ snm. La orientación del cauce principal va de oriente a norponiente, con una longitud del cauce principal de $78 \mathrm{~km}$. Posee un régimen hidrológico nivo-pluvial, presentando una precipitación media anual, entre 1965 y 2000, de $1.801,5 \mathrm{~mm}$.

\section{MATERIAL Y METODOS}

Variables hidrológicas. La información pluviométrica y fluviométrica, entre los años 1965 y 2000 de los ríos Purapel y Achibueno, fue facilitada por la Dirección General de Aguas (DGA), dependiente del Ministerio de Obras Públicas de Chile.

Para el río Purapel, se utilizaron los registros diarios de precipitación de las estaciones Purapel 


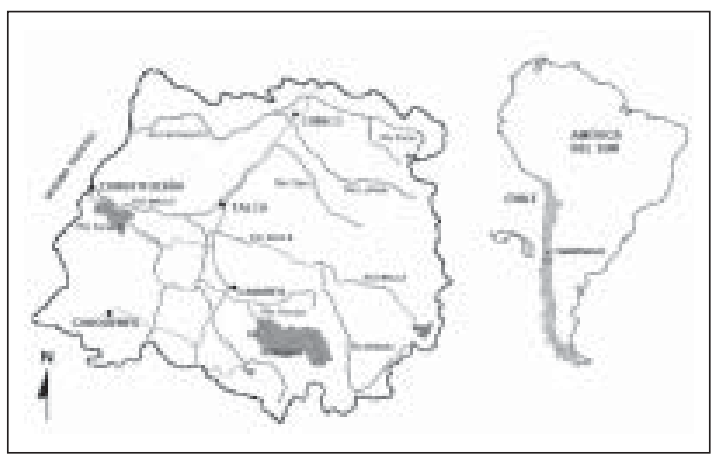

Figura 1. Mapa de ubicación de las cuencas del río Purapel y río Achibueno.

Map of location of the river basins of the Purapel river and Achibueno river.

en Nirivilo y de la estación Constitución. Con estas estaciones se estimaron las precipitaciones medias areales de la cuenca a nivel mensual mediante el método de los Polígonos de Thiessen, dado que Pizarro et al. (7) lo señalan como un método adecuado para la zona. Para la temperatura mensual, se utilizaron los registros de la estación Pencahue, pertenecientes a la Dirección Meteorológica de Chile (DMC). Para los caudales, se utilizaron los registros limnigráficos horarios entre los años 1969 y 2000, con los cuales, y a través de sus curvas de descarga, se construyeron los hidrogramas.

Para el río Achibueno se utilizaron los registros diarios de precipitación de las estaciones Embalse Bullileo y Embalse Ancoa, con los cuales se estimaron las precipitaciones medias areales mediante los Polígonos de Thiessen. Para la temperatura mensual se utilizaron los registros de la estación Embalse Ancoa. Para los caudales se utilizaron los registros mensuales de la estación Achibueno en La Recova entre los años 1965 y 2000.

Los valores de caudal de ambos ríos fueron transformados a aportaciones específicas mediante la expresión [1]:

$$
A_{i}=\frac{Q_{i} * F_{S} * D_{i}}{S}
$$

Donde

$\mathrm{A}_{\mathrm{i}}$ : Aportación específica, para el período “i” (mm/mes).

$\mathrm{Q}_{\mathrm{i}}$ : Caudal medio mensual, para el período "i" $\left(\mathrm{m}^{3} / \mathrm{s}\right)$.
$\mathrm{F}_{\mathrm{s}}$ : Factor de conversión de número de segundos por días y conversión de m a mm (86.400.000).

$\mathrm{D}_{\mathrm{i}}$ : Duración del período "i” en días.

S: Superficie de la cuenca $\left(\mathrm{m}^{2}\right)$.

Modelación matemática. Se calibraron los modelos con dos tercios de las series históricas de datos para cada río, tratando de minimizar la sumatoria del cuadrado de las diferencias entre las aportaciones reales y las aportaciones simuladas por cada modelo (8). De esta forma, el problema de optimización resultó ser el siguiente:

Sea

$A_{r}: a_{r l}, \ldots \ldots \ldots, a_{r n}:$ conjunto de aportaciones reales registradas.

$A_{c}: a_{c l}, \ldots \ldots \ldots, a_{c n}:$ conjunto de aportaciones calculadas.

$P_{1}, \ldots \ldots \ldots ., P_{n} \quad:$ precipitaciones areales medias.

$E T P_{1}, \ldots \ldots, E_{n}$ : evapotranspiraciones potenciales para la zona.

$p_{1}, \ldots \ldots \ldots . ., p_{n} \quad:$ parámetros del modelo.

$k 1, \ldots \ldots \ldots, k_{n} \quad:$ valores máximos admisibles para los parámetros.

$l_{l}, \ldots \ldots \ldots, l_{n} \quad:$ valores mínimos admisibles para los parámetros.

Entonces, $A_{c}$ depende del valor de los parámetros, dada la función [2]:

$$
A_{c}=f\left(P_{1}, \ldots, P_{n} ; \operatorname{ETP}_{1}, \ldots, \operatorname{ETP}_{n} ; p_{1}, \ldots, p_{n}\right)
$$

Luego, la Función Objetivo [3] queda:

$$
\text { Mín } d^{2}\left(A_{r}, A_{c}\right)=\sum_{i=1}^{n}\left(A_{r i}-A_{c i}\right)^{2}
$$

Sujeto a las restricciones [4] y [5]:

$$
\begin{gathered}
P_{1} \leq k_{1} ; P_{2} \leq k_{2} ; \ldots \ldots \ldots, P_{p} \leq k_{p} \\
P_{l} \geq l_{1} ; P_{2} \geq l_{2} ; \ldots \ldots \ldots, P_{p} \geq l_{p}
\end{gathered}
$$

La validación de los modelos se efectuó con el tercio de datos restantes para cada río. Se comprobó que la variable aportación no tenía una distribución normal ni en el río Purapel ni en el río Achibueno, por lo tanto, para el análisis estadístico de los resultados obtenidos se optó por em- 
plear la prueba no paramétrica U de Mann-Whithney (9), además del coeficiente de determinación $\mathrm{R}^{2}$ (6). De esta forma, el planteamiento matemático de cada modelo es el siguiente:

Modelo T. Propuesto originalmente en 1955 (Thornthwaithe et al., 1955; citado por 1); asume en el suelo de la cuenca una capacidad de almacenamiento de humendad (F). Además, dada una precipitación $\left(\mathrm{P}_{\mathrm{i}}\right)$ y una evapotranspiración potencial $\left(\mathrm{ETP}_{\mathrm{i}}\right)$, el almacenamiento de humedad al final del período viene dado por [6]:

$\mathrm{S}_{\mathrm{i}}=\left\{\begin{array}{cr}\operatorname{Min}\left(P_{i}-E T P_{i}+S_{i-1} ; \phi\right), & \text { si } P_{i} \geq E T P_{i} \\ S_{i-1} * e^{\frac{-\left(E T P_{i}+P_{i}\right)}{\phi}}, & \text { si } P_{i}<E T P_{i}\end{array}\right.$

Se producirá escorrentía $\left(\mathrm{Q}_{\mathrm{i}}\right)$ cuando la humedad total en el suelo exceda su capacidad de almacenamiento, siendo este excedente $\left(\mathrm{T}_{\mathrm{i}}\right)$, el que se expresa por [7]:

$\mathrm{T}_{\mathrm{i}}=\left\{\begin{array}{lrr}\left(P_{i}-E T P_{i}\right)+S_{i-1}-\phi, & \text { si } & S_{i}=\phi \\ 0, & \text { si } & S_{i}<\phi\end{array}\right.$

Entonces, la humedad excedente al final del mes es [8]:

$$
H_{i}=\lambda\left(H_{i-1}+T_{i}\right)
$$

Parte de este excedente constituye escorrentía durante el período, siendo modelado por un parámetro $(\lambda)$, el cual expresa la proporción de agua que permanece en la cuenca. Así, la escorrentía simulada al final del mes es [9]:

$$
Q_{i}=(1-\lambda)\left(H_{i-1}+T_{i}\right)
$$

El modelo así planteado contempla el ajuste de dos parámetros $\Phi$ y $\lambda$.

Modelo Témez. Creado por J.R. Témez en 1977 (citado por 3). Este modelo supone que el perfil del suelo está dividido en una zona superior insaturada, y otra inferior que se halla completamente saturada, asemejando su comportamiento a un embalse subterráneo que desagua en la red superficial. El agua precipitada $\left(\mathrm{P}_{\mathrm{i}}\right)$, se divide en evapotranspiración $\left(\mathrm{ET}_{\mathrm{i}}\right)$ y excedente $\left(\mathrm{T}_{\mathrm{i}}\right)$, cuyo cálculo se efectúa según la siguiente ley [10]:

$$
\mathrm{T}_{\mathrm{i}}=\left\{\begin{array}{ccc}
0, & \text { si } & P_{i} \leq P_{o} \\
\frac{\left(P_{i}-P_{o}\right)^{2}}{\left(P_{i}-\delta_{i}-2 P_{o}\right)}, & \text { si } & P_{i}>P_{o}
\end{array}\right.
$$

Donde,

$\delta=\mathrm{H}_{\max }-\mathrm{S}_{\mathrm{i}-1}+\mathrm{ETP}_{\mathrm{i}}$

$\mathrm{P}_{\mathrm{o}}=\mathrm{C} *\left(\mathrm{H}_{\max }-\mathrm{S}_{\mathrm{i}-1}\right)$

$\mathrm{H}_{\max }$ : Máx. capacidad de almacenamiento de humedad del suelo.

$\mathrm{S}_{\mathrm{i}-1}$ : Humedad del suelo al instante $\mathrm{i}$.

C : Parámetro del modelo.

ETP $_{i}$ : Evapotranspiración potencial entre el instante i.

La humedad en el suelo al final del mes está representada por [11]:

$$
S_{i}=\operatorname{Máx}\left\{0 ;-S_{i-1}+P_{i}-T_{i}-E T P_{i}\right\}
$$

Con una evapotranspiración real igual a [12]:

$$
E T_{i}=\operatorname{Min}\left\{S_{i-1}+P_{i}-T_{i} ; E T P_{i}\right\}
$$

La ley de infiltración al acuífero es [13]:

$$
I_{i}=I_{\max } * \frac{T_{i}}{\left(T_{i}+I_{\max }\right)}
$$

Esta infiltración se convierte en recarga $\left(\mathrm{R}_{\mathrm{i}}\right)$ al acuífero, mientras la otra parte de $\left(\mathrm{T}_{\mathrm{i}}\right)$ va a formar parte de la escorrentía superficial o directa [14]:

$$
E_{i}=T_{i}-I_{i}
$$

La ecuación que modela el comportamiento del acuífero es [15]:

$$
V_{i}=V_{i-1}+R_{i}
$$

Así, la escorrentía modelada al final del mes es [16]:

$$
Q_{i}=\alpha * D_{i} * V_{i}
$$

Donde,

$\mathrm{D}_{\mathrm{i}}$ : número de días del mes i. 
El modelo así planteado contempla el ajuste de 4 parámetros $H_{\max }, C, I_{\max }$ y $\alpha$.

A estos planteamientos originales de los modelos se les realizaron variaciones en la estimación de sus parámetros, calculando los mismos para distintos meses o grupos de meses, flexibilizando así la capacidad de ajuste de los modelos. De esta forma, en el caso del río Purapel, el Modelo Témez quedó con 8 parámetros, mientras que en el caso del río Achibueno el modelo $\mathrm{T}$ quedó con 24 parámetros, lo que podría parecer excesivo, pero son los mismos originales que se buscan para meses o grupos de meses.

\section{RESULTADOS Y DISCUSION}

Los cuadros 1 y 2 muestran los resultados obtenidos en la calibración de los Modelos T y Témez, respectivamente, para las cuencas de los ríos Purapel y Achibueno. Durante la calibración ocurrió el problema de existencia de abundantes mínimos locales, que significa encontrar muchas soluciones óptimas, problema que afecta al algoritmo de calibración y que se conoce como regiones de atracción múltiple $(4,10)$. Así, para encontrar una única solución óptima, se escogió por utilizar numerosas combinaciones de valores ini- ciales en la determinación de los parámetros, seleccionando aquella cuyo valor en su función objetivo resultó mínima (11).

Las figuras 2 y 3 muestran el contraste entre las precipitaciones y el caudal real, y simulado, expresados ambos como aportaciones específicas. En estas figuras se observa cómo los modelos simulan el caudal medio mensual, y cómo esta simulación mayoritariamente subestima los valores en el río Purapel con el modelo Témez y sobreestima los valores con el modelo $\mathrm{T}$ en el río Achibueno.

El cuadro 3 muestra los resultados de las pruebas de bondad de ajuste para el Modelo Témez en la cuenca del río Purapel. Se aprecia que, en términos generales, este modelo explica un $82 \%$ de la variabilidad mensual de la aportación, además de entregar resultados satisfactorios dada la aceptación de la prueba U de Mann-Whitney.

En el cuadro 4 se presentan los resultados de las pruebas de bondad de ajuste del Modelo T en la cuenca del río Achibueno. El test de comparación de muestras U de Mann-Whitney arroja como conclusión que no existen diferencias significativas entre la aportación real y la simulada. Por su parte, el valor del coeficiente de determinación $\left(\mathrm{R}^{2}\right)$ expresa que un $61 \%$ de la variabilidad de la aportación simulada está siendo explicada por el modelo.

\section{CUADRO 1}

Parámetros Modelo T, cuenca del río Achibueno.

Parameters Model T, river basin of the Achibueno river

\begin{tabular}{|lcccccccccccc|}
\hline \multirow{2}{*}{ Parámetro } & Abr & May & Jun & Jul & Ago & Sep & Oct & Nov & Dic & Ene & Feb & Mar \\
\hline \multirow{2}{*}{$\Phi$} & 317,17 & 103,04 & 228,41 & 421,75 & 254,97 & 126,05 & 1 & 33,26 & 41,59 & 600 & 188,93 & 600 \\
$\lambda$ & 0,0001 & 0,6641 & 0,4631 & 0,3333 & 0,5666 & 0,6684 & 0,6883 & 0,6050 & 0,4778 & 0,4410 & 0,4861 & 0,4780 \\
\hline
\end{tabular}

\section{CUADRO 2}

Parámetros Modelo Témez, cuenca del río Purapel.

Parameters Témez Model, river basin of the Purapel river.

\begin{tabular}{|lcccccccc|}
\hline Parámetro & $\mathrm{H}_{\max (\mathrm{h})}$ & $\mathrm{H}_{\max (\mathrm{s})}$ & $\mathrm{C}_{\mathrm{h}}$ & $\mathrm{C}_{\mathrm{s}}$ & $\mathrm{I}_{\max (\mathrm{h})}$ & $\mathrm{I}_{\max (\mathrm{s})}$ & $\alpha_{\mathrm{h}}$ & $\alpha_{\mathrm{s}}$ \\
\hline Valor & 339,2 & 293 & 0,01 & 0,67 & 1.000 & 20,5 & 0,013 & 0,008 \\
\hline
\end{tabular}


BOSQUE 26(2): 123-129, 2005

Aplicación de dos Modelos de Simulación Integral Hidrológica

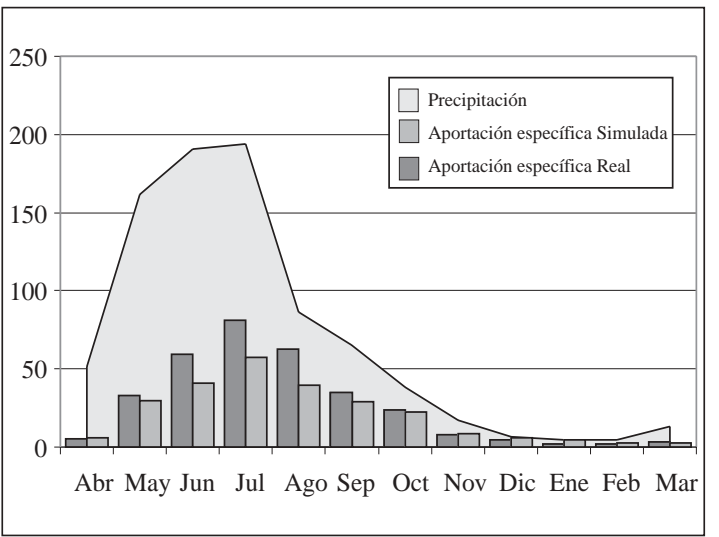

Figura 2. Contraste entre las precipitaciones y las aportaciones específicas reales y simuladas, según el modelo Témez para el río Purapel.

Resistance between the real and simulated precipitations and specific contributions, according to the Témez model for the Purapel river.

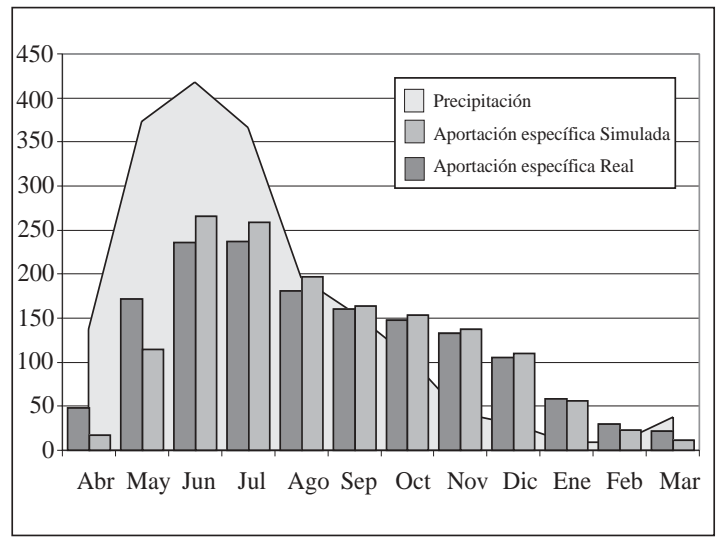

Figura 3. Contraste entre las precipitaciones y las aportaciones específicas reales y simuladas, según el modelo $\mathrm{T}$ para el río Achibueno.

Resistance between the real and simulated precipitations and specific contributions, according to model $\mathrm{T}$ for the Achibueno river.

\section{CUADRO 3}

Pruebas de bondad de ajuste, Modelo Témez, cuenca del río Purapel.

Tests of adjustment kindness, Témez Model, river basin of the Purapel river.

\begin{tabular}{|lccllll|}
\hline \multirow{2}{*}{$\mathrm{N}^{\circ}$ Datos } & \multicolumn{2}{c|}{ Coef. de Determinación $\left(\mathrm{R}^{2}\right)$} & & \multicolumn{2}{c|}{$\mathrm{U}$ de Mann-Whitney } \\
\cline { 2 - 3 } \cline { 5 - 6 } & Calib. & Valid. & & Estadístico & Ut $\quad$ Conclusión \\
\hline 136 & 0,77 & 0,82 & & 0,13 & $\pm 1,96$ & Se acepta Ho \\
\hline
\end{tabular}

\section{CUADRO 4}

Pruebas de bondad de ajuste, Modelo T, cuenca del río Achibueno. Tests of adjustment kindness, Model T, river basin of the Achibueno river.

\begin{tabular}{|lcclccc|}
\hline \multirow{2}{*}{$\mathrm{N}^{\circ}$ Datos } & \multicolumn{2}{c}{ Coef. de Determinación $\left(\mathrm{R}^{2}\right)$} & & \multicolumn{2}{c|}{$\mathrm{U}$ de Mann-Whitney } \\
\cline { 2 - 3 } \cline { 5 - 6 } & Calib. & Valid. & & Estadístico & Ut & Conclusión \\
\hline 192 & 0,71 & 0,61 & & 0,48 & $\pm 1,96$ & Se acepta Ho \\
\hline
\end{tabular}

Las figuras 4 y 5 muestran las gráficas de aportaciones reales y simuladas de la etapa de validación para las cuencas de los ríos Purapel y Achibueno, construidas en cada caso con los modelo Témez y T, respectivamente. Así, se aprecia la relación que guardan las aportaciones reales respecto de las simuladas y cómo estas últimas se asemejan presentando fluctuaciones estacionales. Para el río Purapel se observa que la simulación de los caudales máximos siempre se encuentra por debajo de los caudales reales, siendo subestimativo para estas situaciones. 
BOSQUE 26(2): 123-129, 2005 Aplicación de dos Modelos de Simulación Integral Hidrológica

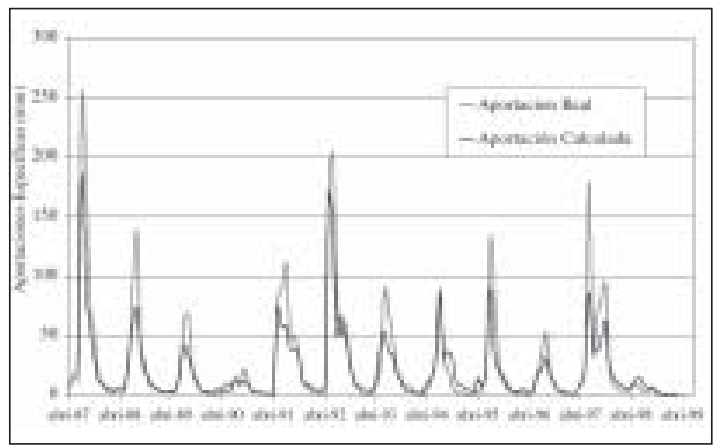

Figura 4. Aportaciones específicas reales y simuladas, Modelo Témez, cuenca del río Purapel.

Real and simulated specific contributions, Témez Model, river basin of the Purapel river.

\section{CONCLUSIONES}

Los Modelos de Simulación Integral de Cuenca (MSIC), Modelo Témez (de 8 parámetros) y Modelo $\mathrm{T}$ (de 24 parámetros) presentan aceptables niveles de ajuste. El Modelo Témez explica un $81 \%$ de la variabilidad de la aportación mensual en la cuenca del río Purapel, mientras que el Modelo T, un $61 \%$ de la aportación de la cuenca del río Achibueno.

En la cuenca del río Achibueno, después de probar los dos Modelos de Simulación Integral de Cuenca, fue posible apreciar que aunque el Modelo T presentó los mejores resultados no fue capaz de modelar o reproducir ciertas etapas del ciclo hidrológico, como las precipitaciones en forma de nieve y los deshielos que ocurren en primavera.

La aplicación de modelos con una mayor cantidad de parámetros no necesariamente refleja una mejor calidad de validación, como lo demuestran los coeficientes de determinación $\left(\mathrm{R}^{2}\right)$ en la cuenca del río Achibueno, lo que determina la necesidad de analizar una mayor cantidad de datos y experimentar sobre el tipo y número de parámetros a utilizar.

Si se considera que los Modelos de Simulación Integral de Cuenca poseen la facultad de estimar series de caudales a partir de datos meteorológicos, resulta de gran utilidad contar con modelos ajustados, los cuales permiten completar series históricas de caudales y generar series en cuencas que no poseen datos, transformándose en una importante herramienta en la planificación de faenas forestales, ya que permiten el diseño de obras hidráulicas asociadas a la evacuación de agua, como puentes, alcantarillas y canales de evacuación. Además, de permitir comparar el com-

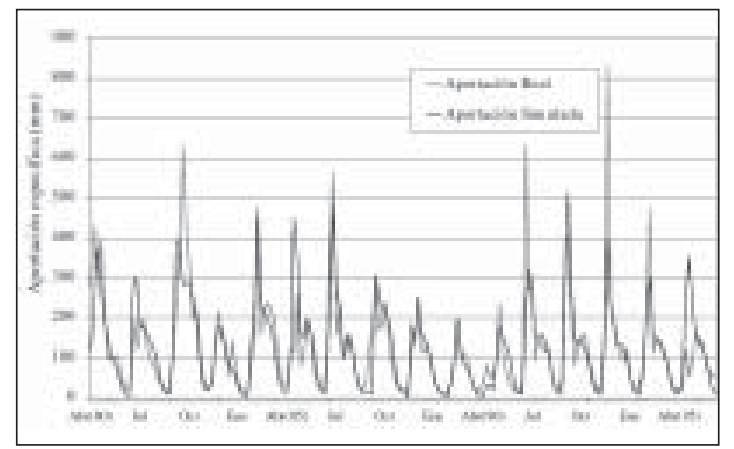

Figura 5. Aportaciones específicas reales y simuladas, Modelo T, cuenca del río Achibueno.

Real and simulated specific contributions, Model T, river basin of the Achibueno river.

portamiento que presentan los caudales en distintas condiciones de cubierta vegetal.

\section{BIBLIOGRAFIA}

(1) LAW, A., D. KELTON. Simulation Modeling and Analysis. U.S.A: McGraw-Hill. 1991. 759 p.

(2) LINSLEY, R., M. KOHLER, J. PAULHUS. Hidrología para Ingenieros. Colombia: McGraw-Hill. 1977. 386 p.

(3) CARMONA, A., J. AYUSO, J.L. AYUSO. Estudio de algunos modelos determinísticos lluvia-escorrentía. Aplicación a una cuenca real. Ingeniería Civil (España), 1990, $\mathrm{N}^{\mathrm{o}} 77$, p. 13-28.

(4) ESTRELA, T. Los modelos de simulación integral de cuencas y su utilización en estudios de recursos hídricos. Ingeniería Civil (España), 1990, No 72, p. 83-95.

(5) RUIZ, J.M., T. ESTRELA, L. QUINTAS. Modelización hidrológica distribuida en el proyecto Guadiana. El modelo SIMPA 1.0 (Sistema Integrado Modelación Precipitación-Aportación). España. 1996.

(6) LLAMAS, J. Hidrología General, Principios y Aplicaciones. España. Servicio Editorial Universidad del País Vasco. 1993. $635 \mathrm{p}$.

(7) PIZARRO, R., C. RAMIREZ, J.P. FLORES. Análisis comparativo de cinco métodos para la estimación de precipitaciones areales anuales en períodos extremos. Bosque (Chile), 2003, vol. 24, No 3, p. 31-38.

(8) SCHWAR, R. Calibration of a conceptual rainfall-runoff model for flood frequency estimation by continuous simulation. Water Resource Research, 1996, vol. 35, $\mathrm{N}^{\circ} 10$, p. 3103-3114.

(9) MASON, R., D. LIND. Estadística para Administración y Economía. USA. Editorial Alfaomega. México. 1995.911 p.

(10)THYER, M., G. KUCZERA, B. BATES. Probabilistic optimization for conceptual rainfall-runoff models: A comparison of shuffled complex evolution and simulated abbealing algorithms. Water Resources Research, 1999, vol. 35, No 3, p. 767-773.

(11) YE, W., B.C. BATES, N.R. VINEY, M. SIVAPALAN, A.J. JAKEMAN. Performance of conceptual rainfall-runoff models in low-yielding ephemeral catchment. Water Resource Research, 1997, vol. 33, No 1, p. 153-166.

Recibido: 12.09 .03 Aceptado: 08.03.04 